\title{
Breast milk against coeliac disease
}

\author{
L M Sollid
}

Should we advise mothers to continue breast feeding while gluten is introduced into their babies' diets?

C oeliac disease is a multifactorial disorder developing as a result of a complex interplay between genetic and environmental factors. ${ }^{1}$ HLA and non-HLA genes are involved, and gluten is obviously a critical environmental factor as the disease goes into remission when gluten is eliminated from the diet. The important case control study by Ivarsson et al concludes that feeding with breast milk when gluten is introduced in children under the age of two years protects against coeliac disease. Breast milk can therefore be considered an environmental factor for this disease as well.

Proving causality using epidemiology is demanding. ${ }^{2}$ There may be both recognised and unrecognised confounding factors associated with breast feeding, which are not properly adjusted for. The authors controlled for one obvious factor-gluten intake. Introducing large amounts of gluten increased the risk of coeliac disease compared with small or medium amounts. Notwithstanding, multivariate analysis demonstrated that breast feeding at the time of gluten introduction in itself reduced the risk of coeliac disease. A plausible mechanism would strengthen the evidence for a causal relationship. What could then be the mechanisms for this protective effect?

The lactating mammary gland and the infant intestine is an immunological dyad. ${ }^{3}{ }^{4}$ Human milk contains defence factors such as secretory IgA antibodies, free secretory component, lysozyme, and lactoferrin which protect the infant's mucosal surfaces. This protection is particularly important in the "window" before the child's own immunity is established. Additionally, the milk contains cytokines and leucocytes which may influence the infant immune system in more sophisticated ways, potentially having long term effects on the child's immune system. ${ }^{3}$ It is poorly understood how the latter is brought about and how important are such "imprinting" effects. The fact that breast feeding protects from intestinal infections is however indisputable.

Basically, the gut immune system has two modes of response to foreign antigen. It may establish a tolerising-type immune response, which is often termed oral tolerance. This response is, depending on the amount of antigen fed,

Ivarsson A, Hernell $\mathbf{O}$, Stenlund $\mathbf{H}$, et al. Breast-feeding protects against celiac disease. Am J Clin Nutr 2002;75:914-21.

Background: While the importance of both genetic predisposition and exposure to dietary gluten in the causation of coeliac disease is beyond question, the recent epidemic of coeliac disease in children in Sweden has highlighted the role of other factors, including other aspects of infant feeding.

Question: Does breast feeding and/or delayed introduction of dietary gluten protect children from developing coeliac disease?

Design and methods: A population based case control study covering the whole of Sweden and involving 627 incident cases of coeliac disease in children (<15 years) and 1254 controls matched for age, sex, and area selected from the National Population Register. Mailed questionnaires were returned by $96 \%$ of cases and $90 \%$ of controls.

Results: The risk of coeliac disease was reduced in children aged $<2$ years $180 \%$ of the cases) if they were still being breast fed when dietary gluten was introduced (adjusted odds ratio (OR) $0.59,95 \%$ confidence interval (Cl) $0.42-0.83$ ). This effect was more pronounced in infants who continued to be breast fed after gluten was introduced (OR $0.36,95 \% \mathrm{Cl} 0.26-0.51)$. The risk was greater when gluten was introduced into the diet in large amounts (OR $1.5,95 \% \mathrm{Cl} 1.1-2.1$ ) than when introduced in small or medium amounts. In older children these risk factors were of little or no importance. Discontinuing breast feeding at the time of introduction of gluten was estimated to account for $31 \%$ of cases and $45 \%$ when combined with large amounts of flour intake.

Conclusion: The gradual introduction of gluten containing foods into the diet of infants while they are still being breast fed reduces the risk of coeliac disease in early childhood and probably also during the subsequent childhood period.

characterised by deletion of reactive $\mathrm{T}$ cells or activation of regulatory $\mathrm{T}$ cells. This response mode may be considered the default reaction of the gut immune system. The alternative response is aggressive in nature, and beneficial for the fighting of viruses and bacteria. The immediate environment in which the immune response is established is thought to play a decisive role in dictating the responding mode ${ }^{6}$; products of bacteria, viruses, or injured cells will activate the antigen presenting cells, which in turn will act on $\mathrm{T}$ cells by expression of costimulatory molecules and cytokines. An aggressive immune reaction will ensue under such priming conditions.

Coeliac disease is the result of an inappropriate immune response to gluten in the gut. ${ }^{1}$ The response is a delayed hypersensitivity reaction, dominated by the $\mathrm{T}$ cell production of interferon $\gamma$. The $\mathrm{T}$ cells that respond to gluten recognise primarily gluten peptides modified (deamidated) by the enzyme tissue transglutaminase (TG2). ${ }^{7}$ A critical question is how and when deamidated gluten peptides are generated. TG2 is naturally involved in the formation and repair of the extracellular matrix. ${ }^{8}$ The enzyme is induced by tissue destruction and inflammation. Gut infection will increase TG2 expression and this will favour the generation of deamidated gluten peptides. ${ }^{1}$ At the same time this will be an environment that instructs the gut immune system to aim for an aggressive reaction. Thus an infection probably functions as a trigger initiating the harmful gluten perpetuated $\mathrm{T}$ cell response of coeliac disease. The simplest model to explain a protective effect of breast milk in coeliac disease would be through the milk's protection against gut infections. More sophisticated models can be envisaged, for example activation of cytokine or idiotype networks by milk constituents. The principle of Occam's razor however speaks in favour of the former.

Epidemiological studies have indicated infections as another environmental risk factor for coeliac disease. In fact, a study of Swedish children demonstrated that the risk for coeliac disease diagnosed before two years of age is significantly higher in children born during 
the summer compared with those born during the winter. ${ }^{9}$ Notably, those born during the summer have gluten introduced into their diet during the winter when infections are more prevalent. Also, case control data indicated that coeliac subjects experienced three or more infection episodes significantly more often than the referents. ${ }^{9}$ These observations fit with the immunological model outlined above.

So what advice should we give to mothers? There is indeed a plausible explanation why continued breast feeding at the time of introduction of gluten protects against coeliac disease although it can be questioned whether the available data are sufficient to prove beyond doubt that this is causal. On the other hand, it is hard to envisage negative factors associated with breast feeding. Hence on balance, it seems justified to advise mothers to continue breast feeding while they gradually introduce gluten to their babies' diets.

Gut 2002;51:767-768

Aun...............

Author's affiliation

L M Sollid, Institute of Immunology, University of Oslo, Rikshospitalet, N-0027 Oslo, Norway;

I.m.sollid@labmed.vio.no

\section{REFERENCES}

Sollid LM. Molecular basis of celiac disease. Annu Rev Immunol 2000; 18:53-81.

2 Hill AB. The environment and disease: association or causation? Proc R Soc Med 1965;8:295-300.
3 Hanson LA, Ceafalau L, Mattsby-Baltzer I, et al. The mammary gland-infant intestine immunologic dyad. Adv Exp Med Biol 2000;478:65-76.

4 Brandtzaeg P. The secretory immunoglobulin system: regulation and biological significance. Focusing on human mammary glands. Adv Focusing on human mammary gl
Exp Med Biol 2002;503:1-16.

5 Weiner HL. Oral tolerance: immune mechanisms and treatment of autoimmune diseases. Immunol Today 1997; 18:335-43.

6 Matzinger P. An innate sense of danger. Semin Immunol 1998;10:399-415.

7 Molberg $\varnothing$, McAdam SN, Körner R, et al. Tissue transglutaminase selectively modifies gliadin peptides that are recognized by gut-derived T cells. Nat Med 1998:4:713-17.

8 Aeschlimann D, Paulsson M.

Transglutaminases: protein cross-linking enzymes in tissues and body fluids. Thromb Haemost 1994:71:402-15.

9 Persson LA, Ivarsson A, Hernell O.

Breast-feeding protects against celiac disease in childhood-epidemiological evidence. Adv Exp Med Biol 2002;503: 1 15-23. 